\title{
Chronic stressors increased susceptibility to colds
}

Cohen S, Frank E, Doyle WJ, et al. Types of stressors that increase susceptibility to the common cold in healthy adults. Health Psychology 1998 May;17:214-23.

\section{Question}

What is the relation between acute and chronic life stressors and susceptibility to the common cold?

\section{Design}

Cohort study in which participants were given a low infectious dose of rhinovirus (type RV39 or Hanks) and quarantined for 1 day before and 5 days after exposure.

\section{Setting}

Pittsburgh, Pennsylvania, USA.

\section{Participants}

276 people (55\% women, $81 \%$ white) aged $18-55$ years who responded to a newspaper advertisement and were free of disease (based on examination and laboratory testing). Exclusion criteria were pregnancy, currently lactating, or on regular medication.

\section{Assessment of risk factors}

Acute and chronic life stressors based on scores from the Bedford College Life Events and Difficulties Schedule. Acute events lasted $<1$ month and were a marked or moderate long term threat (eg, getting reprimanded at work). Chronic stressors lasted $\geqslant 1$ month, did not end $>6$ months before assessment, and were a high moderate or marked long term threat (eg, ongoing unemployment); chronic physical illness stressors were not included. Other variables included health practices (smoking, exercise, sleep habits, alcohol use, and vitamin $\mathrm{C}$ and zinc intake), endocrine and immune function, social network ties, personality characteristics, and control variables (age, sex, race, education, body mass, baseline antibody levels, season, and virus type).

\section{Main outcome measures}

Main outcome was having a cold, based on objective criteria for infection (isolation of virus or changes in serum antibody levels) and illness (mucous weights or mucociliary nasal clearance).

\section{Main results}

$84 \%$ of participants showed evidence of infection, but only $40 \%$ had colds. 179 participants $(65 \%)$ had $\geqslant 1$ acute event in the previous year and 75 participants (27\%) had chronic stressors. Analyses adjusted for control variables showed that participants who had chronic stressors in the previous year were more likely to get colds than those who did not (table); participants who had acute events in the previous year were not more likely to get colds (adjusted odds ratio [OR] 1.4, 95\% CI 0.76 to 2.55). Certain health practices (smoking, exercising $\leqslant 2$ times/wk, poor sleep efficiency, $\leqslant 1$ alcoholic drink/d, $\leqslant 85 \mathrm{mg}$ vitamin $\mathrm{C} / \mathrm{d}$ ), hormone concentrations (increased norepinephrine concentrations), personality factors (introversion), and social network ties (low network diversity) were also associated with an increased risk of colds. The relation between chronic stressors and colds persisted after controlling separately for health practices, endocrine and immune markers, personality, and social network diversity (table).

\section{Conclusion}

People who had chronic severe life stressors lasting $\geqslant 1$ month had increased susceptibility to colds compared with people who did not have chronic life stressors, but people who had acute stressful life events in the previous year did not have increased susceptibility.

Odds ratios for developing a cold for participants with chronic stressors lasting $\geqslant 3$ months compared with those without chronic stressors

\begin{tabular}{lll}
\hline Variables & Adjusted odds ratios* & 95\% CI \\
\hline Chronic stress (CS) & 2.9 & 1.39 to 5.88 \\
CS adjusted for health practices & 2.2 & 1.02 to 4.61 \\
CS adjusted for endocrine and & 2.7 & 1.26 to 5.70 \\
$\quad$ immune markers & 2.7 & 1.30 to 5.60 \\
CS adjusted for personality & 2.6 & 1.27 to 5.48 \\
CS adjusted for social network ties & & \\
\hline
\end{tabular}

*Adjusted for control variables.

Sources of funding: National Institute of Mental Health; National Institutes of Health; Fetzer Institute; John D and Catherine T MacArthur Foundation.

For correspondence: Dr S Cohen, Department of Psychology, Carnegie Mellon University, Pittsburgh, PA 15213, USA. Fax +1 4122683294 .

\section{Commentary}

Interest in the field of psychoneuroimmunology (ie, the study of the role of psychological factors in physical illness) has increased. Much research on the relation between stress and susceptibility to infectious disease has focused on the development of upper respiratory tract infections, which have a fairly high prevalence. Previous studies in which participants were exposed to respiratory viruses showed a relation between psychological stress and the risk of developing a cold. ${ }^{12}$

The goal of the cohort study by Cohen et al was to determine which behavioural and biological pathways link stress to disease susceptibility. The strengths of this study include the use of quarantine both before and after inoculation to control for viral confounders, assessment to rule out disease in participants before study entry, blinded assessment of outcomes, extensive data collection on stressors and potential moderating variables, and use of both subjective and laboratory outcome measures. One weakness is the inclusion of participants who were available for 6 days of quarantine which may make the results less generalisable to a broader population.

Nursing implications include educating people about the linkages between chronic stress and increased risk of infection. Interestingly, this study found that having a work or interpersonal chronic stressor was associated with a greater risk of colds. It is surprising that the study found only a small protective effect of positive lifestyle choices such as exercise and not smoking. The next research step should be to see if interventions aimed at modifying stress can moderate the relation between chronic stress and susceptibility.

Suzanne K Marnocha, RN, CCRN, MSN Assistant Professor, College of Nursing University of Wisconsin Oshkosh, Wisconsin, USA

1 Cohen S, Tyrrell DA, Smith AP. $N$ Engl J Med

1991;325:606-12.
2 Stone AA, Bovbjerg DH, Neale JM, et al. Behav Med 1992;18:115-20. 